\title{
Explications sur la nouvelle loi sur la transplantation et sa mise en application
}

\author{
Le 7 février 1999, le peuple et les chambres fédérales ont approuvé par une \\ grande majorité l'article constitutionnel de la loi sur la transplantation. Envi- \\ ron six ans plus tard, le Parlement a adopté la loi sur la transplantation par le \\ vote final du 8 octobre 2004. La loi sur la transplantation est entrée en vigueur \\ le $1^{\text {er }}$ juillet 2007. Grâce à ceci, la Suisse dispose pour la première fois d'un \\ texte national dans le domaine de la médecine de transplantation. La loi et les \\ ordonnances d'application peuvent être téléchargées sur www.bag.admin.ch/ \\ transplantation. La loi pose le cadre juridique pour la médecine de transplan- \\ tation en Suisse.
}

Correspondance:

Conrad E. Müller MD MBA

Directeur Swisstransplant

Laupenstrasse 37

CH-3008 Berne

Tél. 0313808130

Fax 0313808132

conrad.mueller@swisstransplant.org

www.swisstransplant.org
Sur le plan des transplantations effectuées grâce aux donneurs vivants, nous nous trouvons parmi les meilleurs d'Europe. En revanche, le taux de donneur décédés est inférieur à la moitié de la moyenne européenne (fig. 1). En dehors de la comparaison internationale et malgré des résultats thérapeutiques de transplantation très bons en Suisse, un patient en liste d'attente nécessitant d'urgence un organe décède chaque semaine en raison du manque d'organes.

Nous attendons beaucoup des nouveautés de la nouvelle loi, tout comme de l'introduction standards nationaux; en particulier, un impact positif sur le nombre d'organes disponibles pour le don d'organes.

\section{Embauche et formation \\ de coordinateurs locaux dans tous les hôpitaux disposant de SI}

Une mesure importante et centrale en vue de l'amélioration du travail de donneur est la bonne organisation ainsi que la coordination des processus de transplantation dans les hôpitaux et les centres de transplantation.

Dans tous les hôpitaux équipés d'une station de soins intensifs et dans les centres de transplantation, les cantons sont responsables, en conformité avec la loi, de la définition des processus de transplantation et de l'engagement d'une personne (coordination locale) s'occupant de la gestion et du contrôle de ces derniers (art. 56 de la loi sur la transplantation, art. 45-47 de l'ordonnance sur la transplantation). Les tâches de ce spécialiste comprennent:

- l'identification des donneurs potentiels;
- l'accompagnement des proches;

- la coordination et l'organisation du don d'organes, en collaboration étroite avec la centrale nationale d'allocation des organes, Swisstransplant, www.swisstransplant.org, et les centres de transplantation.

Dans les centres non-transplanteurs, ce poste peut être un poste à temps partiel, couvert par une personne qualifiée de la médecine intensive ou de l'anesthésiologie (médecin ou spécialiste en soins). Les formations de base et continues de ces coordinateurs locaux sont coordonnées dans toute la Suisse annuellement par Swisstransplant et réalisées par les organisations de soutien Les Hôpitaux de Suisse H+, la Foundation to support organ donation FSOD, la Société suisse de médecine intensive SMI et Swisstransplant en collaboration avec les centres de transplantation. Tous les coordinateurs locaux doivent être annoncés à Swisstransplant. Pour des problèmes médicaux lors du travail de donneur, les hôpitaux de périphérie bénéficient du soutien de leur hôpital de référence et d'un conseil médical 24 h/24, 7 j/7 de la part de Swisstransplant.

\section{Consentement explicite élargi} pour la transplantation d'organes

Concernant le prélèvement d'organes, de tissus et de cellules en cas de mort, le principe du consentement explicite élargi est valable: le prélèvement est uniquement admis, si un accord (par exemple une carte de donneur) peut être fourni. Si le désir du défunt n'est pas connu, ce sont les proches qui peuvent prendre une déci- 
Figure 1

Evolution du don d'organes en Suisse de 1990 à 2006. Source: Swisstransplant.
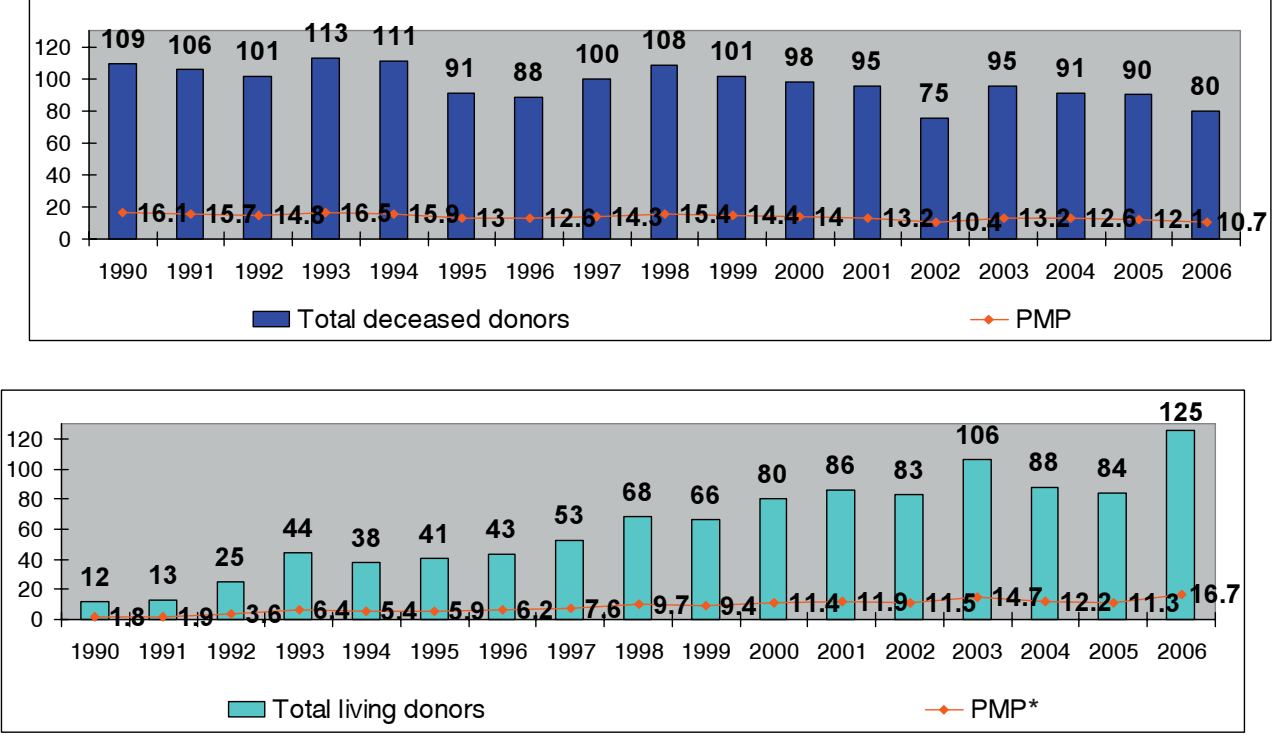

*PMP: per million population.

sion, correspondant à la volonté la plus probable du défunt.

\section{Critères élargis concernant le don vivant}

Chaque personne responsable et capable de discernement qui a été informée sur les avantages et désavantages d'un don de manière complète, peut décider librement et pour sa personne, si elle désire encourir les risques d'un don vivant au profit d'une tierce personne. Une relation familiale ou un lien émotionnel restreint entre le donneur et le receveur ne sont pas requis.

\section{Application des critères de morts basés sur les directives ASSM}

La loi s'appuie selon les critères de mort sur le concept de la «mort cérébrale», d'après lequel un être humain est mort lorsque les fonctions de son cerveau, y compris du tronc cérébral, sont anéanties (directives de l'ASSM de 2005 «Diagnostic de la mort dans le contexte de la transplantation d'organes»; www.assm.ch/ethique).

\section{Allocation centralisée des organes}

L'allocation des organes est effectuée selon la loi sur la transplantation toujours de manière centralisée et exclusivement centrée sur le patient par la centrale de coordination nationale, Swisstransplant. Les critères d'allocation définis juridiquement sont les suivants: l'urgence médicale, l'utilité sur le plan médical, le temps d'attente, et l'égalité des chances. A l'aide d'un programme d'allocation informatisé nouvellement développé, les données du donneur et du receveur sont comparées et le receveur le plus approprié est proposé.

\section{Obligation d'annoncer les patients qui nécessitent une transplantation}

Le médecin traitant d'un patient est tenu, avec l'accord de ce dernier, d'annoncer immédiatement une indication médicale de transplantation au centre de transplantation. Cette annonce doit également être effectuée lorsqu'une thérapie de remplacement, par exemple la dialyse, est menée. Du côté du donneur, tous les hôpitaux et centres de transplantation sont tenus d'annoncer à la centrale de coordination nationale toutes les personnes décédées qui remplissent les critères pour un don d'organes. Les cantons sont tenus d'en informer les médecins en droit d'exercer et les hôpitaux.

\section{Information du public par l'Etat et les cantons / cartes de donneurs}

La loi prévoit que l'Etat et les cantons informent régulièrement le public sur l'importance de la médecine de transplantation. L'information doit être explicitement neutre concernant la question du don. L'information s'adresse en premier lieu à la population, puis aux médecins et au personnel de soins. 
L'Office fédéral de la santé publique a démarré en collaboration avec Swisstransplant au moment de la mise en application de la nouvelle loi sur la transplantation une première campagne d'information: entre autre, l'envoi dans tous les ménages d'une brochure comprenant une carte de donneur et l'ouverture d'un portail internet (www.transplantinfo.ch, www.trapiantinfo.ch). Les hôpitaux et les cabinets de médecins sont également livrés en cartes de donneur. Des cartes supplémentaires peuvent être commandées auprès de Swisstransplant (www.swisstrans plant.org). Toutes les anciennes cartes conservent leur validité.
Si toutes ces nouveautés sont appliquées avec un engagement unanime, il est certain que les temps d'attente de nos patients en liste d'attente vont diminuer et que le résultat général de la médecine de transplantation va être encore amélioré.

Une étude de cohorte soutenue par le Fonds national suisse, la «Swiss Transplant Cohort Study», va régistrer les résultats de transplantation d'organes en Suisse prospectivement à partir de 2008. Nous espérons pouvoir communiquer des résultats positifs dans les années à venir. 